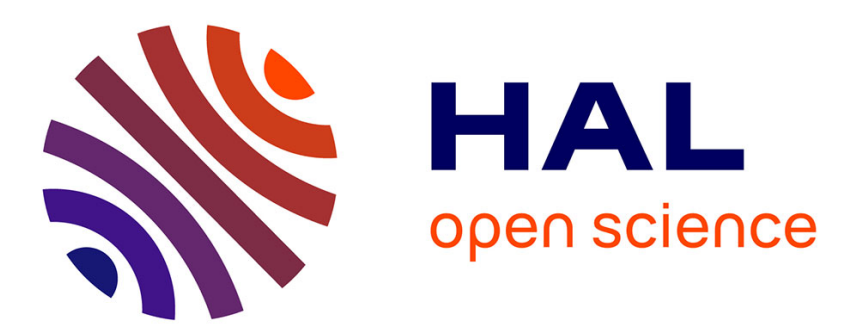

\title{
Une logique de l'autoconservation. Psyché et précarité. Réflexions sur le cadre et le contre-transfert
}

Houriya Abdelouahed

\section{To cite this version:}

Houriya Abdelouahed. Une logique de l'autoconservation. Psyché et précarité. Réflexions sur le cadre et le contre-transfert. Cliniques méditerranéennes, 2005, Précarité, exclusion, abandon, 2 (72), pp.17-28. 10.3917/cm.072.0017 . hal-01508552

\section{HAL Id: hal-01508552 \\ https://hal.science/hal-01508552}

Submitted on 19 Jun 2017

HAL is a multi-disciplinary open access archive for the deposit and dissemination of scientific research documents, whether they are published or not. The documents may come from teaching and research institutions in France or abroad, or from public or private research centers.
L'archive ouverte pluridisciplinaire HAL, est destinée au dépôt et à la diffusion de documents scientifiques de niveau recherche, publiés ou non, émanant des établissements d'enseignement et de recherche français ou étrangers, des laboratoires publics ou privés. 


\section{Houriya Abdelouahed}

\section{Une logique de l'autoconservation Psyché et précarité Réflexions sur le cadre et le contre-transfert}

Dans l'un de ces lieux où le regard est happé par la vue de ce qu'il ne peut soutenir, où le visible vient d'emblée se désigner comme ce qui peut mesurer et juger de la gravité des troubles psychopathologiques, avant même que l'écoute ne puisse saisir des bribes ou des pans entiers de ce qu'il en est des difficultés d'être, dans l'un de ces lieux, je reçois un patient dont je tairai le nom pour des raisons de confidentialité. Extrêmement négligé, un sourire inqualifiable, il me dit venir en consultation sur le conseil de l'assistante sociale qui " s'occupe de lui ». D'ailleurs, continue-t-il, ce n'est pas logique qu'elle s'en occupe autant. Son désir de l'insérer se heurte à son immobilité à lui, qui au demeurant, est désespérante pour elle et " ce n'est pas logique ». De cette " absence de logique », il sera longuement question : qu'à son âge, il continue à vivre chez ses parents (il a 35 ans), qu'il dorme autant, qu'il reste suspendu au système d'aides sociales, qu'il déserte toute activité... En l'écoutant parler de cette "logique-illogique », je lui pose, à mon grand étonnement, cette question : "Qu'est-ce qui n'est pas logique dans votre naissance? » Sa surprise est de taille. Sa mimique change brusquement. Et après un long silence, il relate ce qui ne parvient pas à avoir un sens pour lui, ce qui demeure non logique. Le septième d'une fratrie nombreuse, il porte le prénom d'un frère décédé, prénom qui signifie, en arabe, l'immortel ; l'immortel comme figure inarticulable du désir parental, dirait S. Leclaire. 
Souvent, face à une difficulté de mise à mort ou d'un travail de deuil, les parents confient au second le soin d'immortaliser le premier. Seulement ici, c'est au quatrième né après le décès de celui qui fut désigné comme l'immortel, qu'échoit le commandement d'être le consolateur et le substitut vivant de celui dont l'ombre ou le fantôme continua à rôder tant que les trois garçons nés après sa mort étaient voués à poursuivre leur chemin sur les sentiers de l'existence. Et c'est au quatrième né que revient la tâche d'annuler la disparition. Est-ce logique? De la mort, il sera longuement question. Son état de santé - juste après la naissance - nécessitait une longue hospitalisation. Les médecins étaient formels, sentence à l'unanimité : "Il ne survivra pas. » Mais il survécut. Son père relatant cette histoire ponctue ainsi : "Les médecins disaient tous que tu allais mourir, et tu n'es pas mort, ce n'est pas logique. » Pendant son hospitalisation, la mère n'allait jamais le voir. Depuis, il dort, dit-il. On ne prend pas la peine de le réveiller. Et, il ne fera que « la moitié d'une scolarité ».

Ces événements de vie seront relatés sans la moindre émotion. Il ne se plaint pas. Le ton n'est pas celui des lamentations. Ni tristesse, ni chagrin. Tout semble forclos : le plaisir aussi bien que la douleur. Lentement, il dit l'immobilisme, le manque de vie, le froid. Un froid qui l'interroge plus qu'il ne le préoccupe. Mais il dit ne s'interroger que pendant les séances de psychothérapie. "C'est seulement ici que je pense à tout ça. » Alors que le travail psychothérapeutique est engagé, il relate un événement récent. Il a rencontré sa mère - dame âgée, épuisée par les aléas de la vie et par un grand nombre de grossesses et d'accouchements - portant un lourd panier. Il l'a regardée. Pendant la séance, cette pensée surgit : «Je n'ai pas eu pitié d'elle. Je n'ai rien senti. Je ne l'ai pas aidée. Je l'ai regardée et rien. »

Le travail thérapeutique auprès de ce patient va ouvrir sur cette figure autoconservatrice de la mort et met en évidence cette " mise en conservation du vivant » (P. Fédida) sous une forme inanimée. Le potentiel inné, qui devient - grâce aux interactions de la mère avec l'environnement - continuité d'être, a rencontré ce que Winnicott nomme «l'autre terme d'alternative », à savoir « la réaction qui interrompt cette continuité d'être et l'annihile 1 ». Dans la parole du patient, la rencontre avec la mère - comme bouclier ou comme pare-excitations - fit défaut. Et loin de panser la blessure d'une telle brèche, la parole du père n'offrait pas de cadre pour qu'un désir de vie puisse s'inscrire.

1. D.W. Winnicott, «La théorie de la relation parent-nourrisson », dans De la pédiatrie à la psychanalyse, Paris, Payot, 1969, p. 369. 
«Celui qui augmente sa science, dit l'Ancien Testament, accroît sa douleur. » Afin que la douleur ne le transperce pas, pour ne pas être foudroyé par " l'empiétement de l'environnement»(Winnicott), pour se conserver, ses potentialités psychiques se sont gelées. Et, pour demeurer vivant, l'enfant devait rester inanimé sous l'effet d'une glaciation le privant de toute animation psychique, ce que F. Tustin nomme : la stratégie de «la conservation des émotions par le froid». Ainsi, celui qui est sans vie, ne risque pas de mourir. " Pour mourir, dit justement le patient, il faut vivre d'abord. »

Le voyant confiné dans son immobilité, l'assistante sociale - chargée du suivi social pour le maintien du RMI (revenu minimum d'insertion) - tentaitelle désespérément de réanimer celui qui choisit pour sa conservation le gel psychique ? Était-ce un travail de réparation ? Le patient mobilisait-il chez l'assistante sociale un désir d'enveloppement ?...

Le social achoppait sur une psyché en souffrance.

\section{LE SOCIAL ET LA PSYCHÉ}

La loi du premier décembre 1988 mettant en place le dispositif du RMI (revenu minimum d'insertion) et stipulant une politique dite d'insertion tant sociale que professionnelle, s'est heurtée à ce qu'elle n'avait pas prévu, à savoir l'importance du facteur santé comme entrave à la démarche d'insertion. Les " acteurs du terrain ", se rendant compte de l'insuffisance des réponses pragmatiques et la difficulté à identifier clairement la problématique des personnes ou leurs demandes, commencèrent à faire appel à des «psys » (les psychiatres dans un premier temps, les psychologues ensuite) afin d'évoquer la lourdeur des dossiers d'un nombre considérable de bénéficiaires du RMI croisant ainsi deux sphères de compétences et d'interventions que sont le champ social et le médical. Tracer la limite et la légitimité des pratiques des uns et des autres devait nécessairement, pour ce faire, passer par la définition de la nature du lien entre précarité sociale et précarité psychique. La prise en charge allait de pair avec la définition de l'objet à prendre en charge.

Depuis l'instauration du dispositif du RMI, des plumes vont se mobiliser afin de tenter de cerner " un sujet sensible ou objet trouble » (Fabrice Dhume). Les écrits sociologiques vont, d'abord, s'atteler à la tâche de définir le lien entre modernité et précarité. Celle-ci, écrit Alain Touraine, nous laisse devant la béance « des dissociations par quoi se définit la modernité 2 ». Crise car un principe d'articulation et d'intégration de divers domaines de la vie 
personnelle et collective a disparu, continue A. Touraine, " et il ne semble remplacé par aucun autre. Chacun de ces domaines, laissé à lui-même, se libère ou se dégrade selon le niveau social de ceux qui y participent 3 ».

Cette dégradation sera interprétée en termes de désocialisation, exclusion, rupture des liens. Et la crise de la modernité sera définie comme crise du lien. C'est ainsi qu'on lira La fabrique des exclus ${ }^{4}$, comme un appel de l'auteur au rétablissement du lien. Des travaux historiques et sociologiques relisent Histoire de la folie de Michel Foucault à la lumière des exclus d'aujourd'hui. Mais ce signifiant « exclusion» demeure aussi complexe que problématique. "Douteux », écrit P. Declerck qui s'appuie sur un constat clinique pour récuser la pertinence d'une explication sociologique de la désocialisation car si la clochardisation se résumait à une sorte de victimologie socio-économique, le sujet devrait s'empresser de saisir toute opportunité qui lui permettrait de se rapprocher d'un fonctionnement social plus normal. Or, la réalité clinique est autre ${ }^{5}$.

Constat juste qui permet de reconsidérer l'explication sociologique en termes de victimologie - le social contre le sujet, le sujet affaibli par le social et de réfléchir, par là même, sur le malaise d'un certain discours psychanalytique qui se trouve, à l'instar du sociologique, écrasé sous le poids de la culpabilité et des attitudes surmoïques prêchant par exemple la «fraternité du discours ${ }^{6} »$.

$\mathrm{Au}$ sein d'une littérature psychanalytique naissante, les contributions mettent l'accent sur les effets pathogènes de l'exclusion (sentiments de honte, de culpabilité et de souffrance psychique). Certains écrits cliniques stipulent que l'exclusion nous incite à réfléchir sur les troubles identitaires du sujet exclu, disent en exergue une certaine analogie avec la « symptomatologie des psychoses » avec laquelle ils ne doivent pas se confondre ${ }^{7}$. Reste à savoir en quoi consiste la différence. D'autres écrits insistent sur la complémentarité. Ainsi, nous pouvons lire : «Institution éducative et institution thérapeutique se rejoignent comme lieux préférentiels des enjeux de questionnement et problématisation ${ }^{8}$. » Il $\mathrm{n}^{\prime}$ en demeure pas moins que la nature du questionnement diffère d'un champ à l'autre, d'une institution socio-éducative à une

3. Op. cit., p. 29.

4. J. Maisondieu, La fabrique des exclus, Bayard Éditions, 1997.

5. Cf. P. Declerck, Les naufragés, Plon, 2001.

6. O. Douville, «Incidences subjectives des situations de grande exclusion », dans Le journal des psychologues, octobre 2001, p. 53-57.

7. Y. Gérin, « Le sujet de l'exclusion et la psychologie clinique. Une analyse des perspectives de travail », dans Psychologie clinique, Nouvelle série, n 7, printemps 1999, p. 75-80.

8. M. Audisio, « Précarisations et jeux précaires. Logiques d'exclusion et psychismes », dans Psychologie clinique, op. cit., p. 14. 
« communauté psychanalytique ». On peut également lire au sein de cette littérature, que la rupture fait écho à d'autres ruptures, que l'exclusion fait écho à une autre exclusion : celle de l'individu de/dans sa famille. Dans ce cadre, l'exclusion sera définie comme « la forme comportementale de précaires stabilisations dans le social de certaines économies psychotiques 9 ».

D'abord, cette homogénéisation est loin d'être une réalité clinique. Aussi formulons-nous la même remarque vis-à-vis de P. Declerck qui insiste particulièrement sur la problématique addictive (l'éthylisme). Si les addictés constituent un pourcentage important au sein de cette population, il n'en demeure pas moins que la précarité n'est pas l'apanage des seuls addictés. Comme si l'homogénéisation était une tentative d'inclure lesdits exclus. Ensuite, l'exclusion se trouve être paradoxalement une solide inscription dans un social précaire (instabilité professionnelle, des impayés accumulés, surendettement $\left.{ }^{10} \ldots\right)$.

Cette question de l'inscription est tout à fait fondamentale et va de pair avec celle du lieu. Dans cette sémiotique de l'exclusion, renouer avec la sorcière métapsychologie offre le mérite de poser autrement la question d'un lieu dont l'inscription est exclue. Quel est cet être inapte à l'assimilation ? S'agit-il de ce qui a été exclu ou de ce qui n'est jamais advenu ? Interroger l'inscription et le lieu ouvre sur ce qui demeure encore non suffisamment élaboré, dans une clinique il est vrai difficile, à savoir la question du cadre et du contre-transfert..

\section{UNE INSCRIPTION PROBLÉMATIQUE. TENTATIVE DE LECTURE}

Les séances sont tempêtes ou désert, hurlement ou mutisme. Nous touchons à des zones blessées du narcissisme, à des souffrances identitaires, à des plaintes qui se disent sur le plan ontologique. Le manque à avoir se mêle au manque à être. Le délitement symbolique croise et côtoie le désastre social. Nous sommes pris dans les rets du sensoriel : on est envahi par ce qui se donne à voir (la dégradation physique), par l'olfactif. Et le psychologue qui travaille dans ce champ est confronté non seulement à des problématiques extrêmement lourdes mais à un cadre de travail extrêmement précaire. L.-F. Céline reste d'une grande actualité : le médecin des pauvres est un pauvre médecin. Il en est de même du psychologue qui travaille dans le champ de la précarité lorsqu'on lui demande de pratiquer une clinique « de

9. O. Douville, « Notes d'un clinicien sur les incidences subjectives de la grande précarité », dans Psychologie clinique, op. cit., p. 58.

10. Cf. H. Abdelouahed, "Du trauma, la langue est en souffrance », dans Transhumances IV.

Détresse sociale, souffrance psychique : l'enjeu du sujet, Presses universitaires de Namur, 2003. 
rabais ». Une clinique de rabais alors que nous touchons à la limite, où le sens est aussi opaque que réfractaire, où l'insensé va à l'encontre du travail de sens, jusqu'à défaire la velléité d'interpréter.

Nous ne pouvons alors que montrer notre désaccord face à certaines positions trop confortables : "Dans la mesure où nous cliniciens, face à l'exclusion et à ses incidences subjectives [...] Nous ne sommes pas dépourvus de repères ${ }^{11}$. » Or, la précarité invite le clinicien pour survivre dans son travail clinique, à ne pas rester enfermé dans " la sclérose de ses références " (Ch. David). Et si, comme l'écrit D. Widlöcher, « l'avenir de la psychanalyse est lié à l'extension de ses applications d'une part et la capacité d'innovation et de découverte de la pensée qui l'anime de l'autre 12 », ce n'est pas l'exclusion qui doit être à l'épreuve de la psychanalyse mais le contraire. Quel est l'effet de la précarité sur la théorie analytique ? Par quelles transformations psychiques passe le thérapeute face à ce qu'on nomme " une souffrance qu'on ne peut plus cacher »? Comme si les modifications psychiques produites par le patient sur le thérapeute émanaient avant tout d'un donné sensoriel ? Lorsque le travail thérapeutique est bien engagé, c'est le regard avant l'écoute même qui juge de l'ébauche d'un travail de liaison psychique. Comme si le trouble devait être signifié par ce que le thérapeute capte au niveau de l'olfactif avant que son oreille ne s'ouvre sur le malaise du sujet. Comme si le dysfonctionnement psychique devenait affaire du regard. Le recours actuel à la photographie ne renoue-t-il pas avec cette grammaire visible du trouble mental telle qu'elle s'est constituée à l'orée du XIX ${ }^{\mathrm{X}}$ siècle ${ }^{13}$. La précarité interroge la modélisation métapsychologique : regard/écoute. Piège pour le thérapeute ? Défi pour la situation clinique ? Renouage avec l'observation clinique?

Et si l'errance est un symptôme, elle doit à ce titre être considérée comme " réserve énigmatique de construction et puissance de transformation de modèles métapsychologiques $14{ }$. Afficher une attitude d'autosuffisance visà-vis de ce qui fait symptôme revient à fermer la porte devant aussi bien « l'audace spéculative » (P. Fédida) que devant une inestimable ressource d'observation, d'interrogation et de reproblématisation. C'est se leurrer également sur le véritable sens de la technique. Si l'on suit la définition de P. Fédida sur le sens de la technique - celle-ci n'étant pas seulement le savoir faire mais « la ressource dont l'analyste dispose pour connaître les besoins du

11. O. Douville, op. cit., p. 58-59.

12. D. Widlöcher, «La chose en action », L'inactuel, op. cit., p. 171.

13. Voir par exemple les illustrations qui figurent dans l'ouvrage de P.Declerck, Les naufragés, op. cit.

14. P. Fédida, Crise et contre-transfert, Paris, PUF, 1992, p. 9. 
patient dans les significations vitales les plus élémentaires 15 »- lorsque la béance d'une faille narcissique achoppe sur l'autoconservation, dans quelles ressources puise le thérapeute qui travaille avec les dits déshérités lorsque le besoin d'être aimé se mêle au besoin de se nourrir (être nourri), de se loger (être logé) ?

Si l'on fait nôtre cette formule à savoir que l'exclusion est une inscription solide dans un social précaire, on peut poursuivre que l'errance sociale peut être à la mesure de ce qui erre dans la psyché de par un échec de symbolisation et une incapacité d'inscription. Et ce qui ne fut point admis dans la psyché revient dans le transfert menacer de destructivité et porter atteinte à la situation clinique. Nous pensons à ce que R. Roussillon nomme : le retour des expériences agonistiques " clivées de par leur inaptitude à être symbolisées ». Dans le transfert ces expériences agonistiques reviennent sous forme de destructivité « adressée aussi bien à soi-même qu'à l'environnement actuel 16 ». Ne pas perdre de vue le statut métapsychologique de ce qui erre de par cette inaptitude à la symbolisation est essentiel pour une clinique où le thérapeute est pris dans des stratégies de survie comme thérapeute. Dans un champ où l'on ne cesse de parler de l'économie et de l'économique, le concept économique essentiel qui parait négligé est le contre transfert 17 . Parler de « la fraternité du discours ", au-delà de la séduction politico-idéologique, revient à ignorer ou à méconnaître le négatif, dénier la haine dans le contre-transfert ; la haine comme indice d'un fonctionnement psychique chez le patient et la haine dans le contre-transfert comme réponse émotionnelle et comme indice significatif sur les processus inconscients du patient. Sous couvert du social, on en vient à oublier le fonctionnement psychique du sujet. En outre, «la fraternité du discours », traduit, comme le disait H. Searles, un cramponnement à notre « omnipotence infantile » et un vécu de culpabilité. Laquelle culpabilité rend par moments le thérapeute aveugle devant le sadisme du patient. H. Searles écrit à ce propos : « Le patient contrecarre sadiquement nos efforts pour l'aider et prend un plaisir sadique à nous voir cogner nos têtes contre le mur de sa résistance ${ }^{18}$. »

15. Op. cit., p. 12.

16. Cf. sur ce point R. Roussillon, Agonie, clivage et symbolisation, PUF, 1999, p. 143.

17. Cf. P. Fédida, op. cit.

18. H. Searles, Le contre-transfert, p. 218. Cette question revient inlassablement dans le discours des travailleurs sociaux décrivant des stratégies développées par un certain nombre de RMIstes qui conduisent à l'échec tout projet d'insertion. 


\section{LE TEMPS D'UNE PAROLE}

La question du cadre est liée également à celle du temps. Autre difficulté pour les psychologues et les psychothérapeutes travaillant dans ce champ.

Vers la fin de sa vie, Freud revient dans «L'analyse avec fin et l'analyse sans fin » sur une critique du modèle rankien et écrit : "L'expérience nous a enseigné que la thérapie psychanalytique, la libération d'un être humain de ses symptômes névrotiques, inhibitions et anomalies caractérielles, est un travail de longue haleine ${ }^{19}$. » Et « la tentative de Rank était du reste née de son époque et élaborée sous l'impression du contraste entre la misère européenne de l'après-guerre et la "prosperity" américaine, et destinée à aligner le temps de la thérapie analytique sur la précipitation de la vie américaine ${ }^{20}$. » Mais " théorie et pratique de la tentative rankienne appartiennent aujourd'hui au passé - tout comme la "prosperity" américaine elle-même ». Position que Freud a respectée tout au long de sa vie, puisque dès "Le traitement psychique », il définit le temps comme celui de formation des paroles dans leur pouvoir magique, paroles destinées au traitement psychique, dit : traitement d'âme ${ }^{21}$. Ainsi le silence du thérapeute « correspond au temps qui lui est nécessaire pour laisser se former en lui - à partir de ce qu'il écoute une parole (d'interprétation et de construction) $22 »$. Cette règle qui est exigence de travail, est déniée par le PDI (Plan départemental d'insertion) imposant des thérapies courtes (six mois). Et l'explication freudienne est plus que judicieuse : « De tels efforts n'avaient besoin d'aucune justification, ils pouvaient se réclamer des motivations les plus sensées et les plus appropriées. Mais en eux agissait probablement, un reste de ce mépris impatient avec lequel une période antérieure de la médecine avait considéré les névroses comme des suites superfétatoires de dommages invisibles ${ }^{23}$. »

Dès les premières séances, j'avais senti que le patient objectivait en malaise social une réalité subjective, que le dégel psychique avait besoin de temps, que le désenkystement ne pouvait être que progressif, extrêmement lent. La brutalité d'un réveil pouvait entraîner un agir violent ${ }^{24}$. Respecter le

19. S. Freud, «Analyse avec fin et l'analyse sans fin » dans Résultats, idées, problèmes, II, Paris, PUF, 1985, p. 231.

20. Op. cit., p. 232.

21. S. Freud, «Le traitement psychique », dans Résultats, idées, problèmes, I, Paris, PUF, 1984,

p. 1-23.

22. P. Fédida, Crise et contre-transfert, op. cit., 124.

23. S. Freud, «Analyse avec fin et analyse sans fin », op. cit., p. 231.

24. Beaucoup de psychologues cliniciens et de psychothérapeutes constatent la décompensation sur le plan psychique ou somatique des personnes placées malgré leur fragilité psychique dans des chantiers d'insertion. Dans Des bienfaits de la dépression, P. Fédida explique comment la brutalité d'un réveil peut entraîner une « hémorragie vitale ». Cf. P. Fédida, Des bienfaits de la dépression. Éloges de la psychothérapie, Éditions Odile Jacob, 2001, p. 46. 
temps d'une parole s'ouvrant sur l'inconnu de ce qu'elle va dire revient à mécontenter le PDI. L'exigence clinique achoppe sur l'exigence politicoéconomique.

L'impact des exigences du PDI sur la mobilité associative du thérapeute est de taille. Il est extrêmement difficile d'évaluer les modifications ou la traversée psychique du thérapeute ou sa capacité de résonance face à des patients difficiles. L'angoisse vient-elle des injonctions du PDI ou découle- telle du contact avec la structure psychopathologique des patients difficiles ? L'agir psychique chez le thérapeute vient-il du contact avec la psyché du patient (qui mobilise cet agir) ou de la spécificité de la situation? ou des deux à la fois?

Le thérapeute qui travaille sous l'égide du PDI est terrorisé à l'idée de " perdre un patient ». Cette capacité d'éprouver la perte dont parle $\mathrm{H}$. Searles 25 , dans le cadre d'un travail où l'exigence première ne porte pas sur la nécessité de respecter la structure psychopathologique du sujet ou le respect du temps dont la parole a besoin afin de s'ouvrir sur l'inconnu de ce qu'elle va dire, mais sur un « maintien » qui fait penser à une fantasmatique plutôt anale (garder, retenir, maîtriser...) ; dans ce cadre, la perte renvoie à une spécularité qui affecte la situation clinique. Spécularité car celui qui est censé travailler sur ce qui fait défaut à un travail de liaison et de symbolisation chez le patient se trouve lui-même, quand il ne rentre pas dans le monde de la productivité, parmi les déshérités. Et l'extrême angoisse devant " la perte » rend le thérapeute prisonnier de son moi. L'associativité se trouve d'emblée annulée, ainsi que la dissymétrie qui est la condition et la garante de la situation clinique.

\section{UN TRAVAIL POUR LA PSYCHÉ}

Toute connaissance intériorisée va de pair avec l'investissement du travail, écrit P. Aulagnier 26. Par travail, nous entendons évidemment : travail psychique. Ce dernier amène nécessairement l'évocation d'Éros dont le but consiste en un travail de liaison, travail d'un « je pensant». Un je qui « pour prendre connaissance d'un éprouvé est dans l'obligation de l'interpréter, c'est-à-dire de le lier à une cause qui se nomme désir 27 ». La mise en pensée,

25. H. Searles analyse de façon extraordinairement fine sa confusion lors du déménagement de l'une de ses patientes et écrit notamment : «Tout espoir réaliste non fondé sur le déni doit s'appuyer sur une capacité d'éprouver la perte. »Cf. H. Searles, « Le développement de l'espoir dans la relation patient-thérapeute ", dans Le contre-transfert, Paris, Gallimard, 1979, p. 225-249.

26. P. Aulagnier, "Condamné à investir ", dans Un interprète en quête de sens, Paris, Payot, 2001, p. 243.

27. Op. cit., p. 255. 
comme le rappelle P. Aulagnier, est une mise en sens. Et nous sommes condamnés " pour et par la vie à une mise en pensée et à une mise en sens 28 ». La pensée ou l'activité de penser va de pair avec un travail de liaison et l'investissement de la trace de plaisir. La réalité ne devient humaine que s'il y a ce travail de pensée et de liaison. Mais si le propre d'Éros est de lier, la pulsion de mort consiste à délier. Le désinvestissement, et nous voyons cela régulièrement dans notre clinique, ne porte pas seulement sur un objet mais sur l'acte même d'investissement. Désinvestissement qui menace toute rencontre et toute expérience. Le désinvestissement porte sur la consistance même d'un travail psychique.

Quand ils le peuvent, nos patients racontent progressivement des carences de soins, des expériences traumatiques. Ils disent l'insensé d'une rencontre avec un environnement provoquant le gel psychique ou la faille de l'organisation représentative. Et c'est dans la rencontre transférentielle que s'actualise ce qui n'a pu être lié par la psyché. Ainsi, ce qui n'a pu être pensé ou symbolisé, ce qui reste en souffrance errant dans la psyché, fait retour sous forme de destructivité. Et la répétition des expériences agonistiques (que nous avons déjà évoquées) dans la relation transférentielle est inhérente, comme le dit si bien R. Roussillon, au "processus intégratif ». Ne pas perdre de vue le statut métapsychologique de ce qui erre permet de réfléchir sur ce qui n'est pas advenu du sujet. "La fraternité du discours » implique, donc, une symétrie (toi mon frère je t'aime) qui annule la situation clinique, celle-ci se voit réduite à un réseau d'aide social qui « ligote " le thérapeute ${ }^{29}$. Le malaise social ne doit pas occulter la nécessité du maintien de la négativité qui caractérise la situation clinique. Et du moment où l'on s'engage à faire un travail clinique, il est important de se souvenir de cette parole de Winnicott - surtout lorsque nous travaillons dans le cadre que j'ai tenté de décrire : «On suppose trop facilement que la satisfaction et le sommeil suivent le repas, souvent, c'est le chagrin qui suit cette duperie, surtout si la satisfaction physique prive trop vite l'enfant de son appétit ${ }^{30}$. " Maintenir la dissymétrie, malgré la difficulté de la tâche, revient à ne pas céder sur la négativité de la situation clinique. C'est seulement dans " la tentative orale de la parole » (P. Fédida) que le patient rencontre la séparation de l'objet dont il attend la réponse.

28. Op. cit., p. 238.

29. Nous avons également remarqué que le travail dans ce champ dit de la précarité peut mobiliser des affects d'amour, de compassion, de pitié...

30. D.W. Winnicott, «La position dépressive dans le développement affectif normal », dans De la pédiatrie à la psychanalyse, Paris, Payot, 1969, op. cit., p. 238. Les réponses pragmatiques, disent souvent les travailleurs sociaux, demeurent insatisfaisantes pour un grand nombre de personnes. 


\section{BIBLIOGRAPHIE}

Abdelouahed, H. 2003. « Du trauma, la langue est en souffrance », dans Transhumances IV. Détresse sociale, souffrance psychique : l'enjeu du sujet, Presses universitaires de Namur.

Audisio, M. 1999. " Précarisations et jeux précaires. Logiques d'exclusion et psychismes ", Psychologie clinique, Nouvelle série, $\mathrm{n}^{\circ}$ 7, printemps, p. 11-21.

Assoun, P.-L. 1999. « Le sujet du préjudice : 1'“exclusion” à l'épreuve de la psychanalyse ", dans Dire l'exclusion, Toulouse, érès, p. 15-29.

Aulagnier, P. 1991. "Condamnés à investir », dans Un interprète en quête de sens, Paris, Payot, p. 239-263.

DeClerCK, P. 2001. Les naufragés, Plon.

Douville, O. 1999. "Notes d'un clinicien sur les incidences subjectives de la grande précarité », Psychologie clinique, Nouvelle série, $n^{\circ} 7$, printemps, p. 57-73.

DouvILle, O. 2001. "Incidences subjectives des situations de grande exclusion », Le journal des psychologues, octobre, p. 53-57.

FÉDIDA, P. 1992. Crise et contre-transfert, Paris, PUF.

FÉDIDA, P. 2001. Des bienfaits de la dépression. Éloge de la psychothérapie, Éditions Odile Jacob.

FreUd, S. 1984. «Traitement psychique (traitement d'âme) », dans Résultats, idées, problèmes, I, Paris, PUF, p. 1-23.

Freud, S. 1985. «Analyse avec fin et l'analyse sans fin » dans Résultats, idées, problèmes, II, Paris, PUF, p. 30-268.

GÉRIN, Y. 1999. « Le sujet de l'exclusion et la psychologie clinique. Une analyse des perspectives de travail ", Psychologie clinique, Nouvelle série, $\mathrm{n}^{\circ} 7$, printemps p. $75-80$

MAISONDIEU, J. 1997. La fabrique des exclus, Bayard Éditions.

RoussiLlon, R. 1999. Agonie, clivage et symbolisation, Paris, PUF.

Searles, H. 1979. Le contre-transfert, Paris, Gallimard.

TOURAINE, A. 1997. « La ville coupée en deux », L'inactuel, n 7, printemps, p. 21-31.

WIDLÖCHER, D. 1997. «La chose en action », L'inactuel, n 7, printemps, p. 171-181.

WinNicotT, D.W. 1969. " La position dépressive dans le développement affectif normal », dans De la pédiatrie à la psychanalyse, Paris, Payot, p. 231-249.

WinnicotT, D.W. 1969. " La théorie de la relation parent-nourrisson », dans De la pédiatrie à la psychanalyse, Paris, Payot, p. 358-378.

Résumé

Au lieu d'une lecture sociologique de l'exclusion, l'auteur opte pour une reproblématisation métapsychologique en termes d'inscription et de lieu. De quel lieu s'agitil et quel est cet être inapte à l'inscription ? S'agit-il de ce qui est exclu ou de ce qui n'est jamais advenu du sujet ? Dans la clinique, on remarque que ce qui erre dans la psyché de par une difficulté d'inscription vient répétitivement sous forme de destructivité. Ne pas perdre de vue le statut métapsychologique de ce qui erre permet de reposer différemment la question du cadre et du contre-transfert. Dans un cadre où se 
disent les besoins élémentaires du sujet en termes de : " être nourri » (réactivant ainsi un contre-transfert de nature maternelle), la négativité est la seule garante d'un travail possible. Dire que la satisfaction physique prive trop tôt l'enfant de son appétit, comme l'a formulé Winnicott, revient à définir « la tentative orale de la parole » comme seule possibilité d'advenue du sujet.

Mots-clés

Exclusion, inscription, lieu, psyché, destructivité, sensorialité, cadre, contre-transfert, négativité.

A SELF CONSERVING LOGIC. REFLECTION ABOUT SEETING AND COUNTER-TRANSFERENCE

\section{Summary}

Instead of a sociological reading of exclusion, the author choses to present a new metapsychological problematics which deals with inscription and place. Which place is stake here, and who is it who cannot yield to inscription? Is it what is being excluded, or rather that never happened to the subject ? Clinical experience shows that what keeps wandering in the psyche repetitively reappears in a destructive form. If we keep the focus on the metapsychological definition of these wandering elements, we may differently tackle the issues of the therapy framework and transference. When the subject's basic needs are worded in terms of «being fed » (thus provoking a maternal counter-transference), negativity may be the only way to keep working with the patient. If, as Winnicott said, physical satisfaction too early prevents the child from feeling its appetite, the « oral attempt speak » might then be defined as the only way for the subject to realise himself.

Keywords

Exclusion, inscription, place, destructiveness, sensoriality, therapy framework, countertransference, negativity. 